\title{
Robust design of inspection schedules by means of probability boxes for structural systems prone to damage accumulation
}

\author{
Marco de Angelis, Edoardo Patelli, Michael Beer \\ Institute for Risk and Uncertainty, School of Engineering \\ University of Liverpool, UK
}

\begin{abstract}
The design of inspection schedules is a complex optimization problem that requires the reliability to be assessed. The solution to this problem can be found balancing the costs associated to inspection/repair activities against the benefits related to the faultless operation of the infrastructure. The optimization aims at minimizing the total cost, obtained as the combination of maintenance and failure costs, by tuning some design parameters, such as the number, time and quality of inspections. The reliability is assessed making use of probability boxes, i.e. by accounting for both variability and imprecision. The use of probability boxes relaxes the assumption of exact input probability distributions, which is always too strong given that these distributions are very often estimated within a degree of confidence, or elicited from a finite set of experimental data. The optimization problem is formulated as a time-dependent reliability-based optimization problem, where both objective and constraint functions require the evaluation of upper and lower reliability bounds. The solution to this problem represents a real technological challenge, as the reliability assessment by means of p-boxes is a computationally intensive task, which may take up to few days to be completed on last generation processors. In this paper, an efficient and generally applicable numerical technique, which is capable of producing a solution in a very short amount of time ( $\leq 1$ hour), is proposed. The technique combines a forced Monte Carlo simulation method with an optimization strategy, which makes the interval reliability assessment particularly efficient. The efficiency and accuracy of the proposed technique is shown by means of a literature example involving a fatigue-prone weld in a bridge girder.
\end{abstract}

\section{INTRODUCTION}

Preventive maintenance practice can be extremely cost-effective for mitigating damage accumulation of civil infrastructures. In fact, inspection and repair activities may prevent loss of serviceability or even partial collapse. However, making decisions as to whether and when performing inspections is a very complex task, especially on real-scale engineering systems.

The realistic quantification of costs associated to inspections, repair and failure, requires explicit consideration of the unavoidable uncertainties arising from the damage-propagation process, and from the inspection and repair activities. Uncertainties may come from the inherent variability of the damagepropagation process or from the lack of available knowledge about the process itself. P-boxes are used as a comprehensive mean of representing such heterogeneous uncertainties. An uncertainty model defined using p-boxes is quite general and permits to assess the reliability and sensitivity of the computational model to the uncertainty propagation. In other words, the use of p-boxes adds robustness to the reliability analysis making the analyst more aware of the effects of the uncertainties on the model response.

Within the generalized uncertainty model, the design of maintenance activities is an optimization problem that requires the assessment of reliability box. Reliability-based optimization methods, as described e.g. in Jensen (2002), are invoked to solve this problem.

The number, times, and quality of inspections are the design variables of the optimization, whereas the total cost and the failure probability are the objective and constraint functions, respectively. Formulation and solution to time-dependent reliability-based optimization problems are shown in (Patelli, Valdebenito, \& Schuëller 2011) and in (Valdebenito \& Schuëller 2010a).

In this paper, we propose a general methodology for the efficient solution of the time-variant reliabilitybased maintenance optimization problem, where the reliability is assessed by means of p-boxes. No restrictions in terms of number of inspections and number of uncertain parameters can be found. The method- 
ology is derived from the concept of forced Monte Carlo simulation, used to evaluate the availability of plants (Zio \& Marseguerra 2002), and it is exploited to efficiently assess the time-variant reliability conditional to the inspection outcomes, requiring only the execution of computationally inexpensive functions.

Here, Genetic Algorithms are used to drive the global optimization, as the cost and constraint functions are stochastic and therefore, no information about the derivatives can be efficiently used to converge to the minimum. This comes with quite some more numerical burden, which, however, can be significantly alleviated resorting to code parallelization.

\section{RELIABILITY ASSESSMENT WITH PROBABILITY BOXES}

\subsection{Brief introduction to probability boxes}

P-boxes extend the definition of reliability to an interval of possible alternatives, enclosed by a lower and a upper bound. In reliability and risk assessment, pboxes are invoked to represent what in literature is referred to as uncertainty of Type III, which include both aleatory and epistemic uncertainties. Let $\bar{F}$ and $F$ be non-decreasing function from the real line $\mathbb{R}$ into $[0,1]$ and $\underline{F} \leq \bar{F}$ for all $x \in \mathbb{R}$. A p-box is the set of all non-decreasing functions $F: \mathbb{R} \rightarrow[0,1]$, such that $\underline{F}(x) \leq F(x) \leq \bar{F}(x)$.

\subsection{P-box convolution by means of Monte Carlo simulation}

A Dempster-Shafer structure can be seen as the "discrete" equivalent of a p-box and it is key for the reliability assessment of systems. The input-output convolution of p-boxes, in practice, is performed by using Monte Carlo simulation techniques (Kreinovich, Bernat, Borrett, Mariscal, \& Villa 1991). Reliability assessment is, therefore, performed by i) sampling the equivalent D-S structure of the p-boxes, ii) obtaining the output D-S structure, iii) and ultimately estimating the failure probability bounds.

\subsection{Failure probability upper and lower bounds}

Let $\mathcal{G}: \mathbb{R}^{n} \rightarrow \mathbb{R}$ be the system performance function, $\boldsymbol{\theta} \in \mathbb{R}^{n}$ be a vector of p-boxes, and $\Omega_{F}$ be the domain of unacceptable states (or failure domain), such that $\Omega_{F}=\{\boldsymbol{\theta}: \mathcal{G}(\theta) \leq 0\}$. The system performance is evaluated as $g=\mathcal{G}(\boldsymbol{\theta})$. Each focal element, $\underline{\boldsymbol{\theta}}^{\{s\}}$, of the D-S structure is propagated throughout the system, and the corresponding image is obtained as

$\mathcal{G}\left(\underline{\overline{\boldsymbol{\theta}}}^{\{s\}}\right)=[\underline{g}, \bar{g}]^{\{s\}}$

where,

$\underline{g}=\min _{\boldsymbol{\theta} \in \underline{\overline{\boldsymbol{\theta}}}^{\{s\}}} \mathcal{G}(\boldsymbol{\theta}) ; \quad \bar{g}=\max _{\boldsymbol{\theta} \in \underline{\overline{\boldsymbol{\theta}}}^{\{s\}}} \mathcal{G}(\boldsymbol{\theta})$.
The propagation of individual focal elements leads to the failure probability bounds, obtained using the plausibility and belief function as

$$
\begin{aligned}
& \overline{p_{F}}=\lim _{N_{s} \rightarrow \infty} \sum_{\mathcal{G}\left(\underline{\overline{\boldsymbol{\theta}}}^{\{s\}}\right) \cap \Omega_{F} \neq \varnothing}^{N_{s}} m\left(\mathcal{G}\left(\underline{\overline{\boldsymbol{\theta}}}^{\{s\}}\right)\right) ; \\
& \underline{p_{F}}=\lim _{N_{s} \rightarrow \infty} \sum_{\mathcal{G}\left(\underline{\boldsymbol{\theta}}^{\{s\}}\right) \subseteq \Omega_{F}}^{N_{s}} m\left(\mathcal{G}\left(\underline{\overline{\boldsymbol{\theta}}}^{\{s\}}\right)\right) ;
\end{aligned}
$$

where, $m$ is the mass associated to each focal element of the D-S structure. Note that in Eq. 3 the summation is extended to all focal elements whose intersection with the failure domain is not empty, while in Eq.4 the summation is extended to all focal elements that are entirely contained in the failure domain.

Any distribution function contained within the bounding CDFs, even not belonging to any parental distribution model, can be considered for the problem solution. This implies that the failure probability bounds obtained using this approach, are always wider than those obtained from the parametric approach, because a greater set of candidates is searched for. The failure probability bounds are obtained by computing plausibility and belief of the output D-S structure as shown in Eq. 3 and Eq.4.

One major limitation of this non-parametric approach, despite its efficiency, is the difficulty in identifying the input distribution functions that are responsible for the failure probability bounds. This issue is also known in literature as the tracking problem.

\section{OPTIMIZATION OF MAINTANENCE COSTS}

Two main different classes of costs are considered:

- costs due to inspection and repair, $C_{I}+C_{R}$,

- costs of failure, $C_{F}$.

It is assumed that manufacturing costs are deterministic as they are linked to construction and usage of materials. Note that, as pointed out in Valdebenito \& Schuëller (2010b), the costs of repair and failure are obtained as expected values, $E[\cdot]$ as they are obtained from the estimation of repair and failure probability respectively.

\subsection{Costs due to inspections and repair}

The cost due to inspections depends on inspection quality, $q$, and on the inspection times, $\boldsymbol{t}^{\text {insp }}$, and can be expressed as

$C_{I}\left(q, \boldsymbol{t}^{\text {insp }}\right)=c_{I} q \eta\left(\boldsymbol{t}^{\text {insp }}\right)$;

where, $c_{I}$, is a fixed unit cost, and $q$ (see Eq.7) quantifies the quality of inspections. In the Eq. 5 the function

$\eta(t)=\frac{1}{(1+s)^{t}}$ 
is a discount function of discount rate, $s$. Costs due to repair are present only if repair takes place. Hence they depend on the probability of repair, $p_{R}(q, t)$. The probability of repair, is linked to the probability of detecting the damage within an inspection, $P O D$, which in turns depends on the inspection quality, $q$, the level of damage, $D(t)$, and the technique used to spot the flaw. For example, in fatigue-prone metallic components, non-destructive inspection (NDI) techniques can be used. NDI techniques have an associated probability of detection, which can be modeled as

$$
\operatorname{POD}(t)=\left(1-p_{0}\right)\left(1-\mathrm{e}^{q\left(f_{1}-f_{2} D(t)\right)}\right) ;
$$

where, $p_{0}$, is the probability of not detecting a large crack, while $f_{1}$ and $f_{2}$ are constants that depend on the specific NDI technique. The cost of repair can thus be expressed as

$$
E\left[C_{R}\left(q, \boldsymbol{t}^{\mathrm{insp}}\right)\right]=c_{R} E\left[p_{R}\left(q, \boldsymbol{t}^{\mathrm{insp}}\right)\right] \eta\left(\boldsymbol{t}^{\mathrm{insp}}\right) ;
$$

where, $c_{R}$ is a fixed unit cost, and $p_{R}$ is, clearly, a function of the inspection times. Note that in some cases, the unit cost of repair can be very small or sometimes negligible compared to the cost of inspection.

\subsection{Cost of failure}

The cost of failure depends on the quality, $q$, as well as on the state of damage, $D(t)$. Here, failure cost can be expressed as

$$
E\left[C_{F}\left(q, \boldsymbol{t}^{\mathrm{insp}}, t\right)\right]=c_{F} E\left[p_{F}\left(q, \boldsymbol{t}^{\mathrm{insp}}, t\right)\right] ;
$$

where, $c_{F}$ is a fixed unit cost associated with failure, partial collapse, or unavailability, and $p_{F}\left(q, \boldsymbol{t}^{\text {insp }}, t\right)$ is the failure probability, calculated as in the next Section (see Sec.4). Note that the failure probability depends on both the inspection times, $t^{\text {insp }}$, and on the time when the reliability is assessed, $t$, as it will be explained in the next section.

\subsection{Total cost of maintenance}

The total cost of maintenance is

$$
E\left[C_{M}\right]=C_{I}+E\left[C_{R}\right]+E\left[C_{F}\right] .
$$

\subsection{Formulation of the optimization problem}

The maintenance problem can be generally formulated as a constrained optimization problem, where the constraint represents the limit state safety level that the system has to comply with. Here, the following formulation of the optimization problem is considered

$$
\underset{q \in \mathbb{R}^{+}, \boldsymbol{t}^{\text {insp }} \in\left[0, T_{M}\right]^{N}}{\operatorname{minimize}} E\left[C_{M}\left(q, \boldsymbol{t}^{\text {insp }}, t\right)\right]
$$

$$
\text { subject to } p_{F}\left(q, \boldsymbol{t}^{\text {insp }}, t\right) \leq p_{F}^{\text {critic }}
$$

where, $p_{F}^{\text {critic }}$ is determined by a prescribed limit state safety level. The problem of Eq.11 is addressed using the penalty function

$\psi(c)=1-e^{\alpha|\min (0, c)|} ;$

which is a function of the constraint

$c=-\log _{10}\left(p_{F}\left(q, \boldsymbol{t}^{\mathrm{insp}}, t\right)\right)+\log _{10}\left(p_{F}^{\text {critic }}\right) ;$

where, the constraint is satisfied if $c>0$. The problem of Eq. 11 can, thus, be reformulated into an equivalent unconstrained problem, as

$$
\underset{q \in \mathbb{R}^{+}, \boldsymbol{t}^{\text {insp }} \in\left[0, T_{M}\right]^{N}}{\operatorname{minimize}} E\left[C_{M}\left(q, \boldsymbol{t}^{\text {insp }}, t\right)\right]+y \psi(c) ;
$$

where, $y$ is a penalty factor, which value can be chosen knowing the order of magnitude of the minimum value of the objective function.

\section{EFFICIENT MONTE CARLO STRATEGY FOR THE RELIABILITY ASSESSMENT}

The present numerical strategy is derived from the concept of forced MC simulation described in (Zio \& Marseguerra 2002). The strategy is based on the computation of weights, $w$, which account for the probability of detection and can be computed at any inspection time by reusing the results from the same reliability analysis.

\subsection{Time-variant reliability and failure probability assessment}

As the system, $S(t)$, evolves in time, so does the level of damage, $D$, of specific components. The damage can be expressed as a function, $D=D(\boldsymbol{\theta}, t)=D_{\boldsymbol{\theta}}(t)$, of some input parameters $\boldsymbol{\theta}$, that can be used to quantify the level of damage. In this paper, damage manifests as fatigue, and the computational model is governed by the Paris-Erdogan's law (Paris \& Erdogan 1963). Thus, the input vector, $\boldsymbol{\theta}$, includes the initial crack length, the effective stress range, the shape ratio and any other coefficients of the law. The timevariant reliability is obtained via definition of a critical threshold of damage, $d^{\text {thres }}$, as

$r(t)=1-P\left[D_{\boldsymbol{\theta}}(t) \geq d^{\text {thres }}\right] ;$

Both $d^{\text {thres }}$ and $D_{\boldsymbol{\theta}}(t)$ are uncertain quantities with associated probability distribution functions. The timevariant reliability is obtained as

$r(t)=1-\int_{D(\boldsymbol{\theta}, t) \geq d^{\mathrm{thres}}} h(\boldsymbol{\theta}) \mathrm{d} \boldsymbol{\theta} ;$

where, $h$ is the joint density function of the random vector $\boldsymbol{\theta}$. For simplicity, the capacity $d^{\text {thres }}$ has been included in the input vector, $\boldsymbol{\theta}$. By means of the 
Monte Carlo method the time-variant failure probability, $p_{F}(t)$, can be calculated as

$p_{F}(t)=\int_{-\infty}^{\infty} \cdots \int_{-\infty}^{\infty} \mathcal{I}(\boldsymbol{\theta}, t) h(\boldsymbol{\theta}) \mathrm{d} \boldsymbol{\theta} ;$

where, $\mathcal{I}(\boldsymbol{\theta}, t) \in\{0,1\}$ is the indicator function, which is 1 only if $D(\boldsymbol{\theta}, t) \geq d^{\text {thres }}$.

\subsection{Formulation of the maintenance problem}

The maintenance problem requires the evaluation of the reliability, $r(t)$, over the period of time, $t \in$ $\left[0, T_{M}\right]$.

With no inspections $(N=0)$, the reliability can be assessed as in Eq.16 and estimated as in Eq.17. When inspections are considered, i.e. $N>0$, the reliability of the system is conditional to the inspection outcomes. The reliability is a function of two different times: the actual time $t$, when the reliability is assessed, and the inspection time, $t^{\text {insp }}$, when the inspections are performed. In general, i.e. when $N$ inspections are considered, the reliability is given by the conditional probability,

$$
\begin{aligned}
& r(t)= \\
& 1-P\left[D(t) \geq D^{\text {thres }} \mid t_{1}^{\text {insp }}<\ldots<t_{N}^{\text {insp }}<t\right] .
\end{aligned}
$$

The optimal inspection time is naturally between the following two limiting cases. If inspections are performed too early, $\boldsymbol{t}^{\text {insp }}<<T_{M}$, nearly no damage will be found, and hence no repair will take place. As a consequence the reliability will only be improved marginally, or even not improved at all. On the other side, if inspections are done too late, $\boldsymbol{t}^{\text {insp }} \simeq T_{M}$, the probability of detection would be large (because directly related to the level of damage), but it is likely that the system will have already failed, thus, the inspection will not be effective.

\subsubsection{Assumptions}

In order to better illustrate the procedure and without restricting the generality of the approach two simple assumptions are made:

1. Any inspection is followed by only two outcomes: either the flaw is detected or not. If a flaw is detected repair takes place, which action is assumed to be perfect, i.e. after repair $D\left(t>t^{\text {insp }}\right)=0$.

2. Only preventive maintenance is considered. If the critical threshold is exceeded at the time of inspection, the component is not repaired. That is, if failure has occurred, repair actions will not take place.

\subsubsection{Classification of events and total failure probability}

In order to calculate the reliability as defined in Eq.18, mutually exclusive events are classified. Among all four main classes of events are identified:

- the failure event, $F_{i}=\left[D\left(t_{i}^{\text {insp }}\right)>D^{\text {thres }}\right]$, at the time of the $i$-th inspection,

- the failure event, $F_{t}=\left[D(t)>D^{\text {thres }}\right]$, at the evaluation time $t$,

- the repair/detection event, $R_{i}=\left[\delta\left(t_{i}^{\mathrm{insp}}\right)=1\right]$, at the time of the $i$-th inspection,

- the event $\overline{R_{i}}=\left[\delta\left(t_{i}^{\text {insp }}\right)=0\right]$, i.e. the event of nonrepair/non-detection;

where, $\delta \in\{0,1\}$ is a binary random variable to characterize the outcome of inspections, which mass function is

$\delta(t)=\left\{\begin{array}{cc}1 \text { (success) } & \lambda_{D}(t) \\ 0 \text { (failure) } & 1-\lambda_{D}(t)\end{array} ;\right.$

where, $\lambda_{D}(t)=P O D\left(D_{\boldsymbol{\theta}}(t)\right)$ is the likelihood of detecting the flaw during inspection.

The probability of the failure event can be obtained as

$$
\begin{aligned}
& P[F]= \\
& \sum_{j=1}^{N+1} P\left[F_{N-j+2} \cap\left(\overline{F_{N-j+1}}\right)\right] \prod_{k=0}^{N-j+1} P\left[\left(\overline{R_{k}}\right)\right]
\end{aligned}
$$

where, for simplicity, the summation has been extended to $N+1$ to include the failure event at the observation time $F_{t}=F_{N+1}$.

\subsection{An efficient advanced MC simulation approach}

An expression for the estimation, by means of MC simulation, of the failure probability conditional to the inspection outcomes, is derived from Eq.20 as

$$
\begin{aligned}
& \hat{p}_{F}(t)= \\
& \frac{1}{N_{S}} \sum_{s=1}^{N_{S}}\left(\sum_{i=1}^{N}\left(1-\left(1-\delta_{i}^{\{s\}}\right) \mathcal{I}\left(\boldsymbol{\theta}^{\{s\}}, t_{i}\right) \mathcal{I}\left(\boldsymbol{\theta}^{\{s\}}, t_{i+1}\right)\right)\right. \\
& \left.\prod_{k=0}^{i-1}\left(1-\delta_{k}^{\{s\}}\right)+\mathcal{I}\left(\boldsymbol{\theta}^{\{s\}}, t\right) \prod_{k=1}^{N}\left(1-\delta_{k}^{\{s\}}\right)\right) .
\end{aligned}
$$

where, $t_{i}=t_{i}^{\text {insp }}, \delta_{i}=\delta\left(t_{i}^{\text {insp }}\right)$, and the indicator function is

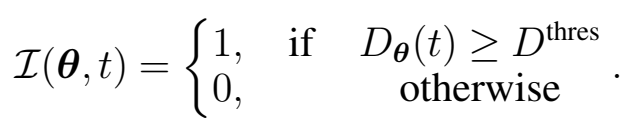


From the expression of Eq.21 follows that a full simulation needs to be performed any time the inspection time vector, $t^{\text {insp }}$, changes, as new inspection outcomes have to be generated. This limitation can be removed noting that the expected value of $\delta(t)$ equals the probability of detection

$$
\begin{aligned}
& w(t)=E[\delta(t)]= \\
& \lim _{N_{S} \rightarrow \infty} \frac{1}{N_{S}} \sum_{s=1}^{N_{S}} \delta(t)^{\{s\}}=E[P O D(D(\boldsymbol{\theta}, t))] .
\end{aligned}
$$

The aforementioned expected value can be used in Eq.21 as a weight to avoid generating new outcomes any time a different inspection time is picked. This will make it possible to use results coming from the same reliability analysis for every selected inspection time. This leads to the estimation of the failure probability as

$$
\begin{aligned}
& \hat{p}_{F}(t)= \\
& \frac{1}{N_{S}} \sum_{s=1}^{N_{S}}\left(\sum_{i=1}^{N} \mathcal{I}\left(\boldsymbol{\theta}^{\{s\}}, t_{i}\right)\left(1-\left(1-\hat{w}_{i}\right) \mathcal{I}\left(\boldsymbol{\theta}^{\{s\}}, t_{i+1}\right)\right)\right. \\
& \left.\prod_{k=0}^{i-1}\left(1-\hat{w}_{k}\right)+\mathcal{I}\left(\boldsymbol{\theta}^{\{s\}}, t\right) \prod_{k=1}^{N}\left(1-\hat{w}_{k}\right)\right)
\end{aligned}
$$

where, this time the samples $\boldsymbol{\theta}^{\{s\}}$ are generated once and for all. An equivalent alternative and more efficient way to compute Eq. 24 without the use of a "for" loop, over the number of samples $N_{S}$, is by means of

$$
\begin{aligned}
& \hat{p}_{F}(t)= \\
& \frac{1}{N_{S}} \sum_{s=1}^{N_{S}}\left(\mathcal{I}\left(\boldsymbol{\theta}^{\{s\}}, t\right) \prod_{j=1}^{N} 1-\hat{w}_{j}\left(1-\mathcal{I}\left(\boldsymbol{\theta}^{\{s\}}, t_{j}\right)\right)\right) .
\end{aligned}
$$

\subsection{Total and partial probability of repair}

By means of the direct approach, the probability of repair can be calculated for the $i$-th inspection as

$p_{R_{i}}=\lim _{N_{S} \rightarrow \infty} \frac{1}{N_{S}} \sum_{s=1}^{N_{S}} \prod_{k=1}^{i} \delta_{k}^{\{s\}}$.

By inverting the order of summation and product sequence, the probability of repair for the $i$-th inspection can be calculated as

$p_{R_{i}}=\lim _{N_{S} \rightarrow \infty} \prod_{k=1}^{i} \frac{1}{N_{S}} \sum_{s=1}^{N_{S}} \delta_{k}^{\{s\}}=\prod_{k=1}^{i} w_{k}$.

The estimator for the total probability of repair, after all inspections have been performed, is obtained using a finite sample set of $N_{S}$ samples as

$\hat{p}_{R}=\prod_{k=1}^{N} \hat{w}_{k}$
Table 1: Mission time and annual frequency

\begin{tabular}{llll}
\hline & Value & Unit & Description \\
\hline$E[\nu]$ & 7.5 & {$\left[10^{6}\right.$ cycles/year $]$} & Annual number of cycles \\
$T_{M}$ & 50 & [years $]$ & Mission time \\
\hline
\end{tabular}

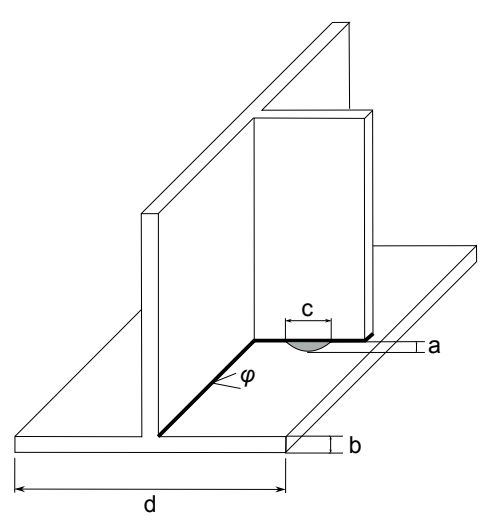

Figure 1: Illustration of structural detail and crack

\section{NUMERICAL EXAMPLE}

In this section a welded connection, taken from $\mathrm{Lu}-$ kic \& Cremona (2001), is under consideration. Welds are particularly weak to damage accumulation as imperfections may form and grow under cyclic loading. The analyzed weld with associated defect is shown in Fig.1. From the picture it can be appreciated that the crack propagates from the weld toe towards the bottom mining the integrity of the structural connection. The crack propagation phenomenon is modelled using the Paris-Erdogan law, by means of

$$
\frac{\mathrm{d} a}{\mathrm{~d} N}=C(\Delta K)^{m}
$$

where, $a$ is the crack length, $N$ is the number of loading cycles, $\Delta K$ is the stress intensity factor range, and $C$ and $m$ are two material parameters. In this study no threshold is put on the $\Delta K$ values, thus, it is assumed that all loading cycles count on damage. Eq. 30 is solved implicitly by means of numerical integration. The stress intensity factor range is a function of the crack length

$\Delta K=Y(a) M(a) \Delta S \sqrt{\pi a}$

where, $Y(a)$ is the stress intensity correction factor, and $M(a)$ is the stress concentration factor, which are also function of the crack shape, $a / c$, the flange thickness, $b$, the flange width $w$, the weld height, $h$, and the weld angle, $\phi$ (see e.g. Figure 1). In order to solve Eq. 30 a target life time (or mission time), $T_{M}$, of the structural component has to be selected, as shown in Table 1. From the rain-flaw histogram (loading analysis) it is possible to derive the annual number of cycles, $\nu$, which multiplied by the target life time, in years, provides the total number of cycles at the mission time. Thus, the number of cycles at the target life time is $N_{\max }=\nu \cdot T_{M}$. 


\subsubsection{Uncertainties}

In this example p-boxes are obtained from given distribution functions considering their distribution moments as bounded real intervals. The parental distribution models are defined for all of those inputs that are known to have a certain distribution, or as Gamma distribution otherwise. The uncertainty model is constructed from the nominal values of mean and standard deviation of Table 2, by adding increasing levels of imprecision. The material quantities $\ln (C)$ and $m$ show a strong negative Gaussian correlation, which is usually close to 0.99 . However, the correlation coefficient is never a precise number and here it is assumed to range in the following bounded interval $\bar{\rho}=[-0.995,-0.935]$. Here, the relative uncertainty factor $e_{I} \in[0,1]$ defines the level of imprecision for the uncertain quantities, so, for instance, the bounded interval of the mean value of quantity $i$ is

$\underline{\bar{\mu}}_{i}=\left[\tilde{\mu}_{i}\left(1-e_{I}\right), \tilde{\mu}_{i}\left(1+e_{I}\right)\right]$;

where, $\tilde{\mu}_{i}$ is the central (nominal) mean value of quantity $i$. In Table 2 , the bounded intervals for mean and standard deviation corresponding to two different levels of imprecision are shown. With the above uncertainty definition, the analyst can relax the assumption of using exact values for the probability distribution definition, which is always too strong at this stage of the analysis. Moreover, by means of uncertainty propagation, it is possible to appreciate the output sensitivity to the amount of uncertainty. This helps recognizing the relative importance of the computational model parameters on the output uncertainty.

\subsection{Failure probability sensitivity}

Self-contained measure of global sensitivity can be extracted from such a general uncertainty model, using pinching. Here, pinching is performed replacing the input quantity with a precise distribution function corresponding to the central values of Table 2 . The sensitivity measure is obtained as

$S_{\theta_{i}}=\left|1-\frac{U\left(\theta_{\sim i}\right)}{U(\boldsymbol{\theta})}\right| ;$

where, $U\left(\theta_{\sim i}\right)$ is the amount of uncertainty outputted pinching parameter $\theta_{i}$, and $U(\boldsymbol{\theta})$ is the total amount of uncertainty. The procedure is repeated for every input quantity, pinching the inputs one by one. The amount of uncertainty is computed as

$U\left(\theta_{\sim i}\right)=\ln \left(\overline{p_{F}}\left(\theta_{\sim i}\right) / \underline{p_{F}}\left(\theta_{\sim i}\right)\right)$.

The global sensitivity analysis was performed choosing 4 different levels of imprecision to better explore the response of the model to the amount of uncertainty. The analysis led to the results shown in Figure 2, where clearly the most important input quantity is the material parameter, $m$, followed by the stress range $\Delta S$, the annual number of cycles, $\nu$, and the material parameter, $C$.

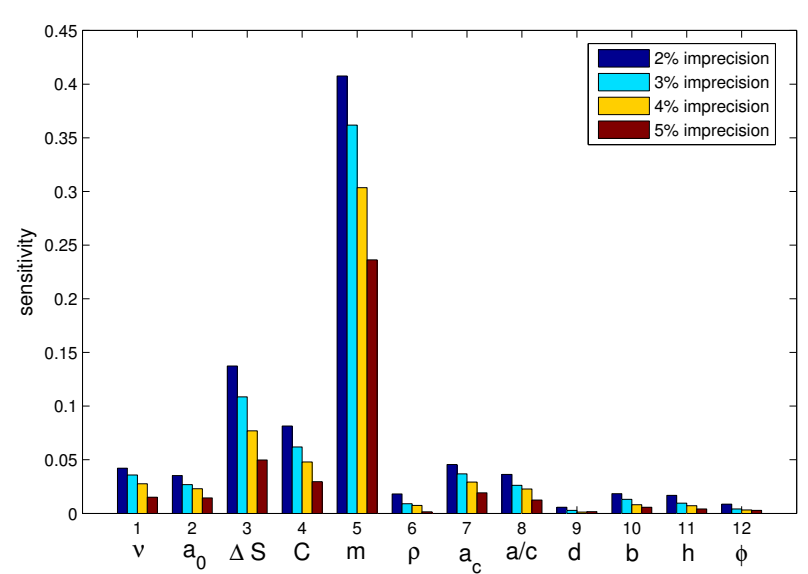

Figure 2: Global sensitivity on the failure probability is obtained pinging one input at a time

Table 3: Unit cost for the numerical example

\begin{tabular}{lll}
\hline Cost & Value & Description \\
\hline$c_{I}$ & 5000 & Unit cost of inspection \\
$c_{R}$ & 500 & Unit cost of repair \\
$c_{F}$ & $5 \cdot 10^{5}$ & Unit cost of failure \\
$r$ & $10^{-3}$ & Discount rate \\
$p_{F}^{\text {thres }}$ & $10^{-3}$ & Failure probability threshold \\
\hline
\end{tabular}

\subsection{Definition of constraint and cost function}

It is recalled that the inspection time vector and the inspection quality are the design variables of the problem. The input unit costs used in this example are reported in Tab.3. In this example the objective is represented by upper bound cost function. Upper and lower bounds of the total cost are displayed in Fig.3, as a function of the first inspection time with fixed inspection quality, $q=1$. From Fig.3, it can be appreciated the influence of just $1 \%$ of imprecision on the solution of the optimization. For the cost upper bound the minimum is located at 27 years, while for the lower bound the minimum is at about 36 years. Equivalently, the failure probability has two different minima, one for the upper and one for the lower bound, as shown in Fig.4. The constraint is defined so that no acceptable solution carries a failure probability upper

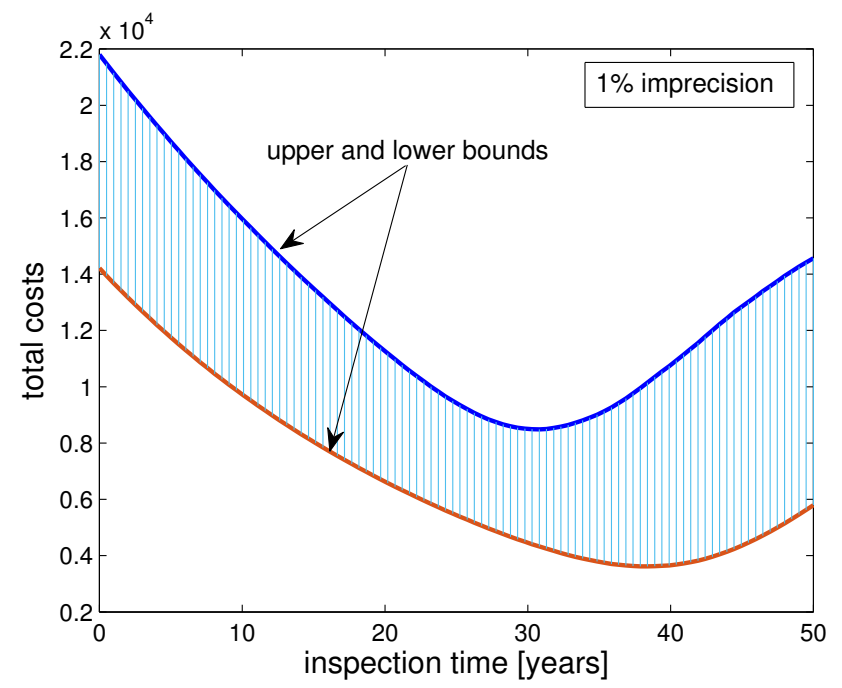

Figure 3: Total cost upper and lower bounds define the set of possible states and candidate solutions 
Table 2: Central values and bounded intervals for the mean and standard deviation of the input p-boxes

\begin{tabular}{|c|c|c|c|c|c|}
\hline \multicolumn{2}{|c|}{ P-box } & \multicolumn{2}{|c|}{ Central values } & \multicolumn{2}{|c|}{ Relative uncertainty: $e_{I}=0.05$} \\
\hline$\theta_{i}$ & Distribution & $\tilde{\mu}_{i}$ & $\tilde{\sigma}_{i}$ & $\underline{\underline{\mu}}_{i}(5 \%)$ & $\bar{\sigma}_{i}(5 \%)$ \\
\hline$\nu\left[10^{6}\right.$ cycles/year $]$ & Lognormal & 7.5 & 0.5 & {$[7.125,7.875]$} & {$[0.475,0.525]$} \\
\hline$a_{0}[\mathrm{~mm}]$ & Lognormal & 0.125 & 0.045 & {$[0.119,0.131]$} & {$[0.043,0.047]$} \\
\hline$\Delta S[\mathrm{MPa}]$ & Gamma & 7.800 & 0.100 & {$[7.410,8.190]$} & {$[0.095,0.105]$} \\
\hline$C^{*}\left[10^{-13} \mathrm{~mm} /\right.$ cycles $]$ & Lognormal & 2.500 & 0.923 & {$[2.375,2.625]$} & {$[0.877,0.969]$} \\
\hline$m^{*}$ & Normal & 3.000 & 0.040 & {$[2.850,3.150]$} & {$[0.038,0.042]$} \\
\hline$a_{f}[\mathrm{~mm}]$ & Gumbel & 1.000 & 0.050 & {$[0.950,1.050]$} & {$[0.019,0.021]$} \\
\hline$a / c$ & Lognormal & 0.400 & 0.160 & {$[0.380,0.420]$} & {$[0.152,0.168]$} \\
\hline$d[\mathrm{~mm}]$ & Gamma & 812.0 & 8.100 & {$[771.4,852.6]$} & {$[7.695,8.505]$} \\
\hline$b[\mathrm{~mm}]$ & Gamma & 31.60 & 3.200 & {$[30.02,33.18]$} & {$[3.040,3.360]$} \\
\hline$h[\mathrm{~mm}]$ & Gamma & 8.400 & 0.700 & {$[7.980,8.820]$} & {$[0.665,0.735]$} \\
\hline$\phi[\operatorname{deg}]$ & Gamma & 35.00 & 2.000 & {$[33.25,36.75]$} & {$[1.900,2.100]$} \\
\hline
\end{tabular}

*Correlation: $\tilde{\rho}(\ln C, m)=-0.99, \quad \bar{\rho}(\ln C, m)=[-0.995,-0.935]$

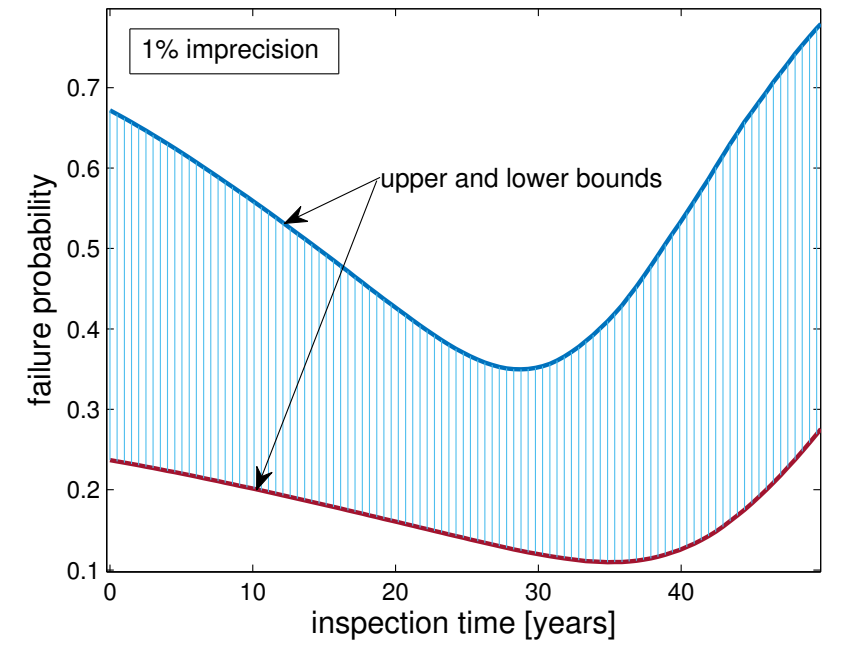

Figure 4: Failure probability upper and lower bounds

bound greater than $p_{F}^{\text {thres }}=10^{-3}$.

\subsection{Time variant failure probability}

The failure probability increases with time as the damage accumulates. However, performing a number of inspections increases the reliability over time. After inspection, the failure probability keeps increasing, but at a much lower rate, as shown in logarithmic scale in Fig.5. In order to better appreciate the scale of failure probability reduction followed by the inspections, the failure probability curves are also shown in Fig.6 in normal scale.

\subsection{Solution to the cost optimization for scheduling multiple inspections}

Results from the constrained optimization with an increasing number of inspections, are obtained fixing the critical failure probability to $p_{F}^{\text {critic }}=10^{-3}$, as also shown in Tab.3. Tab.4 shows that there is no sensible decrease in the total cost of maintenance, as the number of inspections increases. However, the optimum

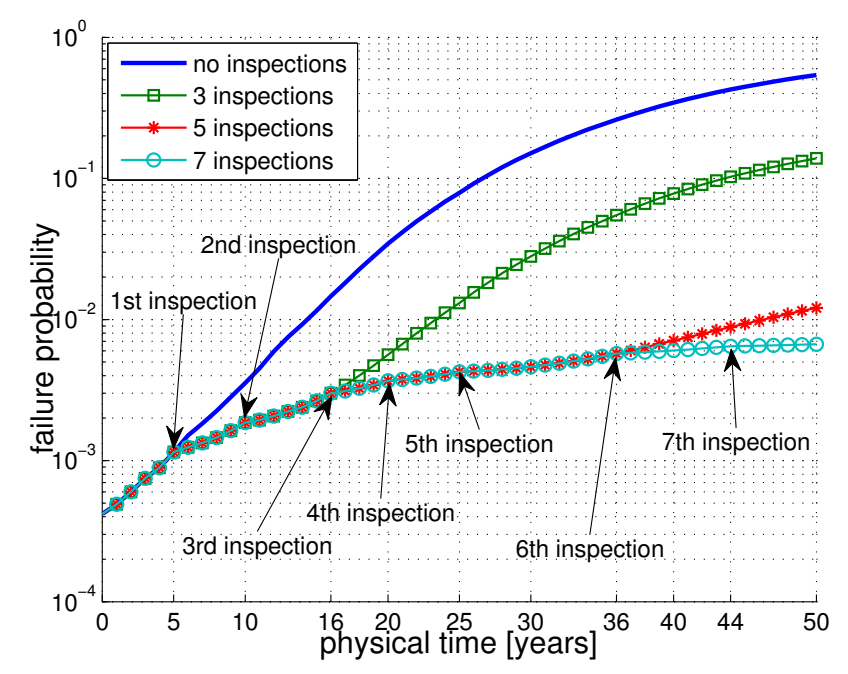

Figure 5: Failure probability curves obtained performing 0, 2, 5 and 7 inspections with fixed quality

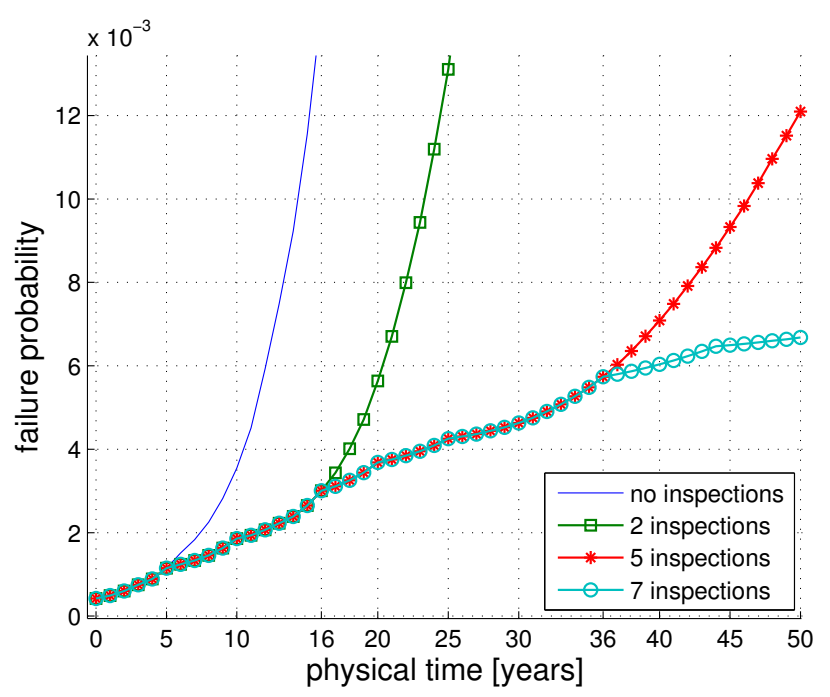

Figure 6: Failure probability curves obtained performing 0, 2, 5 and 7 inspections with fixed quality 


\begin{tabular}{lllll}
\hline $\mathbf{N}$ & $\hat{p}_{F}$ & $\min C_{M}$ & $q^{*}$ & $\boldsymbol{t}^{\text {insp* }}[$ years $]$ \\
\hline 4 & $510^{-1}$ & $3.2710^{5}$ & 0.8 & $\{20,26,28,29\}$ \\
5 & $10^{-3}$ & $3.8610^{6}$ & 88.6 & $\{0.5,7,14,20,30\}$ \\
7 & $10^{-3}$ & $3.7410^{6}$ & 62.0 & $\{0.02,4.5,9,14,20,27,35\}$ \\
9 & $10^{-3}$ & $3.6810^{6}$ & 47.5 & $\{0.02,2,7,11,14,19,24,29,36\}$ \\
12 & $10^{-3}$ & $3.8210^{6}$ & 37.9 & $\{0.02,1.5,4.5,9,11,18,22,26,32,37,45\}$ \\
15 & $10^{-3}$ & $3.8210^{6}$ & 30.3 & $\{0.02,0.5,4,7,9,11,14,16,19,23,25,29,34,39,46\}$ \\
\hline \multicolumn{2}{r}{ * Candidate optima } & & &
\end{tabular}

quality decreases, which means that, at the same total cost, less expensive inspection techniques could be used. Clearly, performing 4 inspections in this case is not enough to meet the safety constraint requirement, as the failure probability is too big, $\hat{p}_{F}=0.5>10^{-3}$, in this case. The last two columns of Tab.4 report the solution of the stochastic optimization problem, in terms of inspection quality and inspection time vector.

\section{CONCLUSIONS}

A general and efficient methodology for the scheduling of multiple inspections has been presented. The maintenance of a system is a challenging engineering task, where the estimation of costs requires the consideration of heterogeneous uncertainties arising from the damage propagation process and from the inspection/repair activities. The methodology makes use of the concept of forced MC simulation to maximize the efficiency, without reducing the accuracy. The efficiency of the MC strategy has permitted to go beyond the classical probabilistic modeling of the uncertainties. The proposed strategy is capable of assessing the failure probability upper and lower bounds, when imprecision is defined in the input probability distributions. In other words, the proposed strategy allows for the definition of the input uncertainties by means of p-boxes, which generalize the concept of probability distribution to include every probability distribution within the two bounding CDFs. In terms of computational cost, only two full reliability analyses are required to assess upper and lower bound at any given time and for any number of inspections. The efficiency, scalability and applicability of the proposed methodology has been demonstrated via an example involving 12 variables. In the example, the advantage of using such a generalized uncertainty model has clearly emerged, as it has been also possible to obtain sensitivity measures on the failure probability, without any additional computational costs. As a future direction, this work can be extended to include interval design variables to see how the uncertainty affects the bounds of the minimum cost.

\section{REFERENCES}

Jensen, H. A. (2002). Reliability-based optimization of uncertain systems in structural dynamics. AIAA journal 40(4), 731738.

Kreinovich, V. Y., A. Bernat, W. Borrett, Y. Mariscal, \& E. Villa (1991). Monte-Carlo methods make Dempster-Shafer formalism feasible.

Lukic, M. \& C. Cremona (2001). Probabilistic assessment of welded joints versus fatigue and fracture. Journal of Structural Engineering 127(2), 211-218.

Paris, P. \& F. Erdogan (1963). A critical analysis of crack propagation laws. Journal of Fluids Engineering 85(4), 528-533.

Patelli, E., M. A. Valdebenito, \& G. I. Schuëller (2011). General purpose stochastic analysis software for optimal maintenance scheduling: application to a fatigue-prone structural component. International Journal of Reliability and Safety 5(3), 211-228.

Valdebenito, M. \& G. Schuëller (2010a). Design of maintenance schedules for fatigue-prone metallic components using reliability-based optimization. Computer Methods in Applied Mechanics and Engineering 199(33), 2305-2318.

Valdebenito, M. A. \& G. I. Schuëller (2010b). A survey on approaches for reliability-based optimization. Structural and Multidisciplinary Optimization 42(5), 645-663.

Zio, E. \& M. Marseguerra (2002). Basics of the Monte Carlo method with application to system reliability. LiLoLe, Hagen. 